\title{
Research on outdoor special effect device for night forest park theme park
}

\author{
You-Suk Kim1, Eun-Seo Song2, Sung-Dae Hong3*
}

1 Reaserch Professor, Seokyeong Unversity, VR Future Center, 16-1 Jungneung-Dong Sungbuk-Ku Seoul, 136704 Korea

2 Reaserch Professor, Seokyeong Unversity, VR Future Center, 16-1 Jungneung-Dong Sungbuk-Ku Seoul, 136704 Korea

$3^{*}$ Professor, Seokyeong Unversity, Dept. of Film and Digital Media, 16-1 Jungneung-Dong SungbukKu Seoul, 136-704 Korea

kimyousuk@gmail.com ${ }^{1}$, songsun1999@gmail.com ${ }^{2}$, sungdaehong@gmail.com*3

Corresponding author ${ }^{*}$ : mobile Phone: +82-010-5218-0267

Article History: Received:11 november 2020; Accepted: 27 December 2020; Published online: 05 April 2021 Abstract: Recently, there have been many forest park theme parks for night tours. Various media technologies are installed in these forest park theme parks. A case study was conducted to find out the purpose and use of various media technologies used in forest park theme parks at night. We divided media technology into three major types of forest park theme parks currently in operation at home and abroad. The case was analyzed by dividing the media technology into forms that used special effects for stage performances, forms with interactive technology, and forms of lighting used for the atmosphere of the theme park. The analysis of the cases with three factors has resulted in the pros and cons of the cases in each part. The advantage is that special effects, interactive technology, and theme lighting are pioneering the media market by bringing out a new type of attraction called the Forest Park Theme Park. The first solution to this was to overcome the difficulties caused by outdoor installation in nature. It was a method to maintain temperature, humidity, dust, etc. in factors such as housing of electronic electrical equipment elements and covers of lighting. Second, we found elements that do not fit in nature. Most of the factors that were not aesthetic to the use of content were artificially installed. To compensate for this, elements such as electroplate ng tubes and camouflage control boxes should be applied from the content design and spatial design stages. By optimizing the essential elements for operation and harmonizing with nature, visitors can minimize visual fatigue and maximize aesthetics. The needs and complements of the design of the forest park theme park studied in this paper deal with the areas that need to be improved for users who have come to the night tour. This is meaningful for the development of a more enjoyable and pleasant forest park theme park.

Keywords: theme park, outdoor light, special effect, stage effect.

\section{Introduction}

Most of the recent special effects of forest park night content have been used outdoors, with stage lighting and special effects devices that have been used only indoors. In the case of stage lighting, it was difficult to produce cases or select installation sites for use in outdoor natural environments, which were designed and used based on indoor concert halls. In many cases, an outdoor type product was used or its case was manufactured and used to handle waterproofing, dustproofing, and heat dissipation for outdoor use. In this paper, we first study the case of stage lighting and special effects used in various night time forest park contents, and through this, we will study a device suitable for forest park contents[1-3].

\section{Case Study}

In this paper, we focused on the examples of Singapore's Rainforest Lumina theme park overseas and the Sonata of Light of Oak Valley Resort and Vivaldi Forest theme park of Daemyung Resort in Korea. Through the case study, the case was analyzed with three perspectives: the application of stage performance technology to the forest park theme park, the installation site with interactive technology, and the lighting that forms the atmosphere of the theme park[4-6].

\subsection{Rainforest Lumina in Singapore}




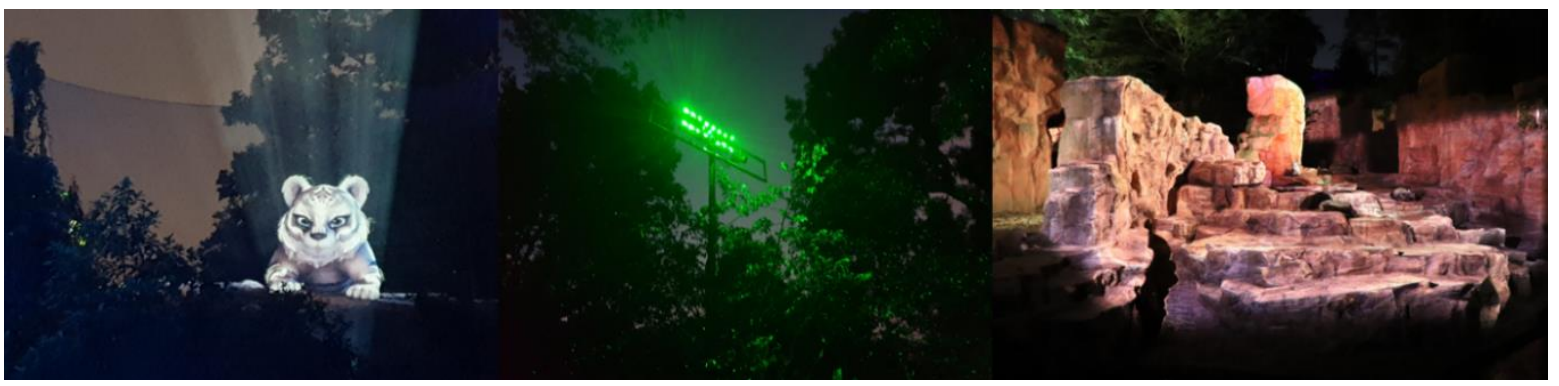

Figure 1. Outdoor use cases for special stage lighting

Figure 1 is the existing special stage effect technology used in outdoor space and is a hologram screen, laser effect, and projection mapping technology in order. These technologies were made using existing indoor equipment as outdoor equipment, and all of them were used as outdoor equipment except for projectors. Advantages of existing special stage effects are applied to outdoor space, which indicates high effect. The hologram screen is divided into rear and front. Two methods are used depending on the location and effect of the installation, divided by the user's point of view and the presence of the background. The laser effect is a very difficult method in outdoor space. If you want to see laser light with your eyes, you need to use a fog machine and a haze machine together, but it is difficult to maintain a certain amount because the wind and temperature change in the outer space. In the case of projection mapping, it can be divided into forest parks by projecting into artificially generated formations or projecting into natural objects. The existing methods of LED media facade (LMF) and projection media facade (PMF) are upper-level concepts[7], and the actual production method and projection method are determined according to the type of artifacts and natural objects. Rainforest Lumina was projected onto a natural-looking artificial stream that was formerly a zoo cage. The audience will see the zoo's cage in a visible form.

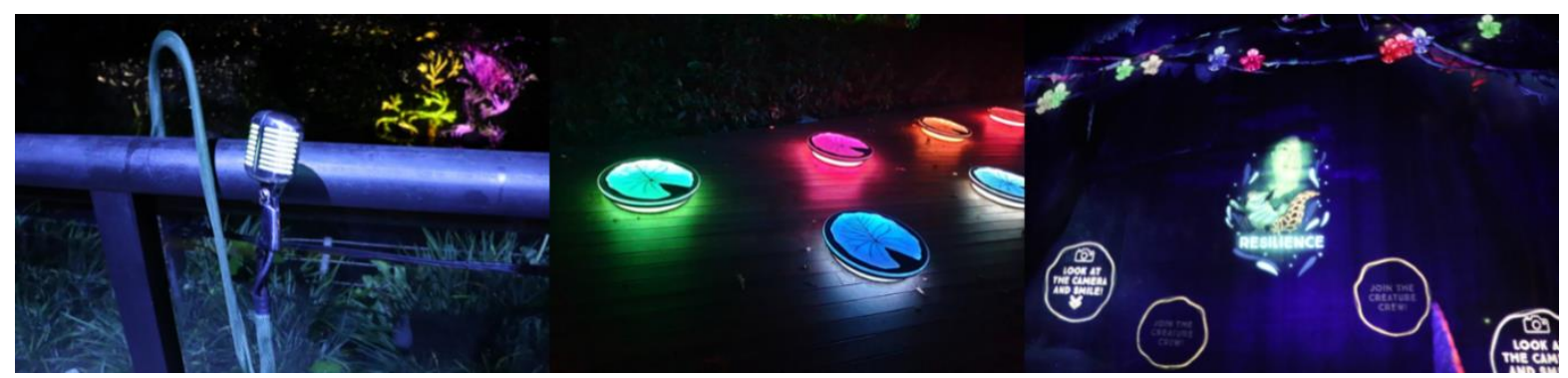

Figure 2. Outdoor interactive special effects

Figure 2 shows the interactive special effects for users of night forest parks. In order, it is a media wall using RFID and webcam, with changing contents according to volume using microphone, footplate lighting using weight sensor, and media wall using RFID and webcam. The use of these new media elements leads to the active participation of users in static, open-circuit effects, enabling a higher level of storytelling. Due to the nature of the forest park theme park, which is frequented by family units, it does not require complicated interaction. It is designed to operate content through relatively simple actions, and changes in projection and lighting are activated as a result. These interactive technologies play a role in allowing visitors to stop for a certain amount of time on the line to spend time. Interactive technologies play an important role in driving time through content consumption during the viewing time of the theme park, which has an overall running time of about an hour. The audience will perform two acts. Number one is to experience by executing a technique, and number two is to record the process. Most of the audience will re-record the results of their actions with their cell phones and others. It repeats actions similar to the mirror effect of early interactive media.

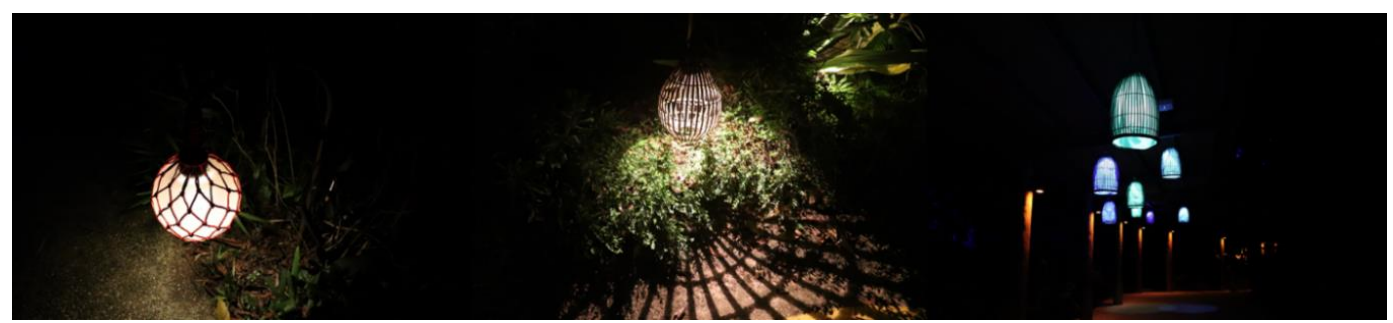

Figure 3. Outdoor lighting fixture design 
Figure 3 is an outdoor lighting fixture design. Each night forest park has a theme. The active reflection of these themes and the use of lighting fixtures suitable for the theme are the mise-en-scene of the entire theme park. It increases the user's immersion in space and also serves as a photo zone. Forest park theme parks located in natural spaces are usually produced under the theme of traditional culture, tales, and stories of space around them. Lighting is the most effective way to form the atmosphere of the space with these narrative elements. The appearance, color, and brightness of lighting are the most important background factors for visitors to appreciate content. Lumina Forest uses lighting fixtures suitable for the atmosphere in each region to create a sense of unity and a partial atmosphere[8].

\subsection{Domestic Night-time Forest Theme Park}

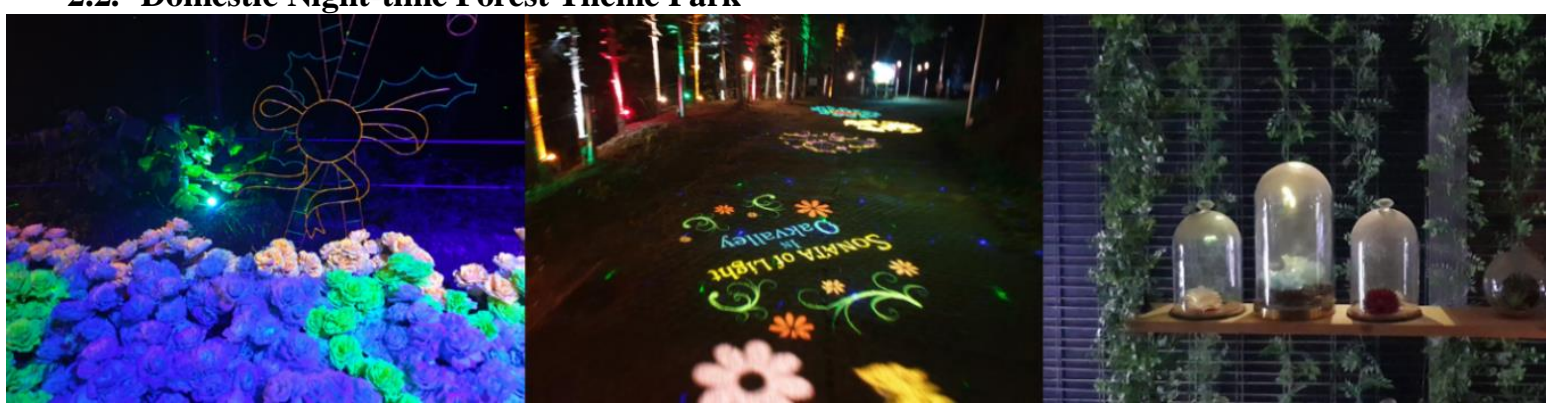

Figure 4. Outdoor use cases for special stage lighting of Sonata of Light

Figure 4 shows the black light, gobo light and miniature hologram technologies among the existing special stage effect technologies used in outdoor spaces. Blacklight is a special lighting using UV light and fluorescence, and unlike the colors seen in visible light areas, it is a fluorescent, heterogeneous color that responds to ultraviolet light and is used in many areas as well as stage performances. Especially in the case of night-time, the effect is strong in places without natural light, so it is mainly used in dark indoor or outdoor spaces at night. The second is a type of lighting that projects a form of penetrating shadow or color filter by placing a screen in front of a strong pin light. Recently, Logo light has been widely used for outdoor advertising and safety phrases. However, on the stage, it is a technology with a long history, and it is a special lighting that creates patterns of various patterns by installing shadow filters in front of long pin lights. It is used in various places due to the development of LED technology, miniaturization of lighting, and development of color picture plate making technology using UV printing. The third is miniature hologram technology. It is also called pseudo holograms and reflective holograms. At first, it used to be mainly used in rectangular boxes with oblique reflectors, but recently, it has been applied to cylinder-type, circular, and square-shaped forms. These miniature holograms are widely used in forest parks at night, as they are cheaper, more transparent, and more effective in applying small theme content than using transparent screens. This technology is more utilized due to the nature of the forest park theme park, which has higher spatial immersion than video playback using displays and aims to harmonize contents in nature as it can be easily applied to the surrounding environment.

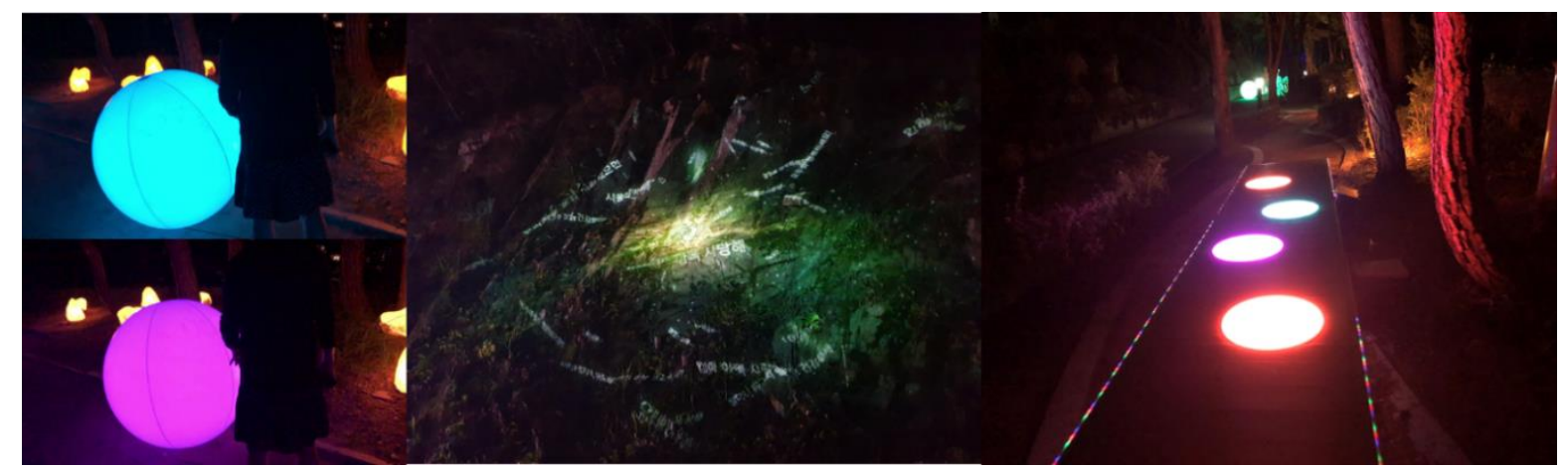

Figure 5. Outdoor interactive special effects of Vivaldi Forest and Sonata of Light

Figure 5 shows touch balls using the interactive technology of the night forest park, text plotting using a mobile phone, and circular lighting that changes when stepped on. Rather than the interactive installation contents of the moment factory that we looked at earlier, domestic interactive installation showed many simple and plain forms. Because this content is installed outdoors, it is somewhat disappointing because there are many things that focus on durability and maintenance. The first is a one-dimensional interaction light in which the light changes when the ball is touched with a touch ball. It is simple, but that is why it has an interactive structure that is easily accessible to the general people. The second is the interactive installation, in which the text you enter appears on the screen projected by your projector and disappears after being rich. It is an 
interactive operation that usually induces the installation of an app using a QR code, or displays face pictures and phrases (name, wish, prayer, etc.) on a large display using an app or web page installed at the time of first entry, and induces the user to take a picture of the screen again. The third is a tread plate-type light that changes when stepped on, similar to the touchball we have seen earlier. It is manufactured using touch, weight, and pressure sensor, and when the user steps on it, it is detected through the sensor and the lighting value installed inside changes. It is relatively simple interactive, but it is easy to draw users' actions, and it is a good interactive element to create a movement line, so it does not fall into the night forest park theme park.

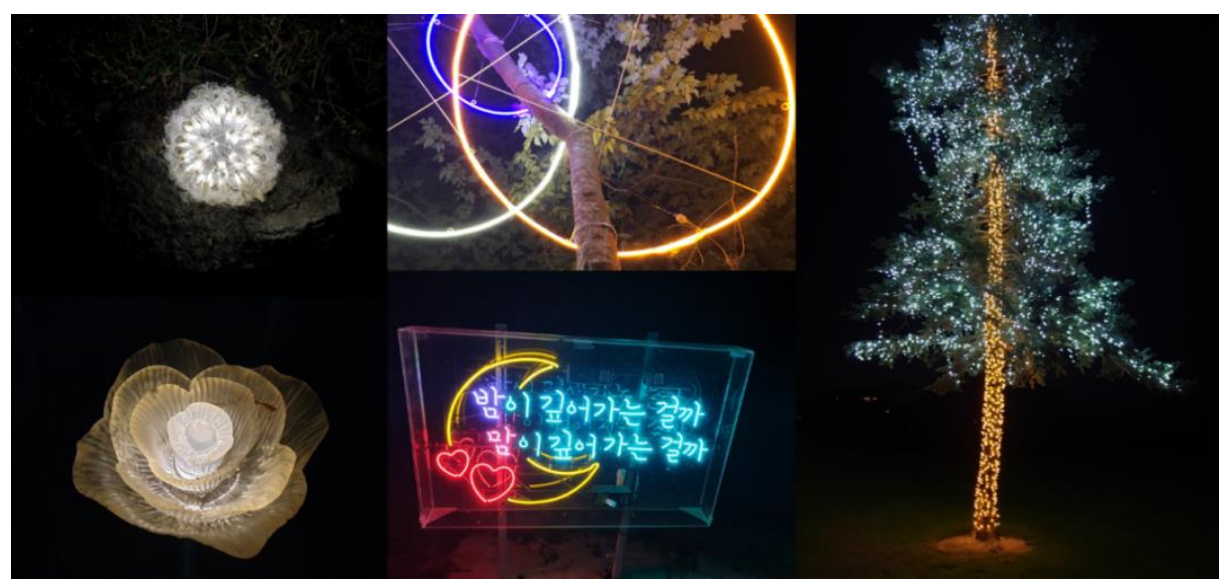

Figure 6. Outdoor lighting fixture design of Vivaldi Forest and Sonata of Light

Since most of Korea's forest parks are based on local traditional tales, many types of lighting are installed to emphasize the beauty of nature. As shown in Figure 6, floral, arboreal, and moon-like forms of lighting are commonly installed in most forest park lighting. In order to easily dazzle the public and to have efficiency due to the nature of the theme park, colorful and photo-friendly lights are placed first rather than feature lighting with a lot of storytelling[9].

\section{Analysis and Derivation}

There are three main special effects used in the night forest park through the case study: existing special stage effect technology, interactive technology, and outdoor lighting fixture design. These technologies require a variety of installation techniques. First, it can be divided into a form of cover and self-type. Cover type is an auxiliary installation of a lighting fixture in the form of covering target equipment, which is designed to use special stage effects devices that are not designed outdoors as outdoor types. The self-type is applied to forest parks by strengthening the exterior frame of lighting fixtures by attaching poles, frames, etc. to install outdoortype special devices[Figure 7].

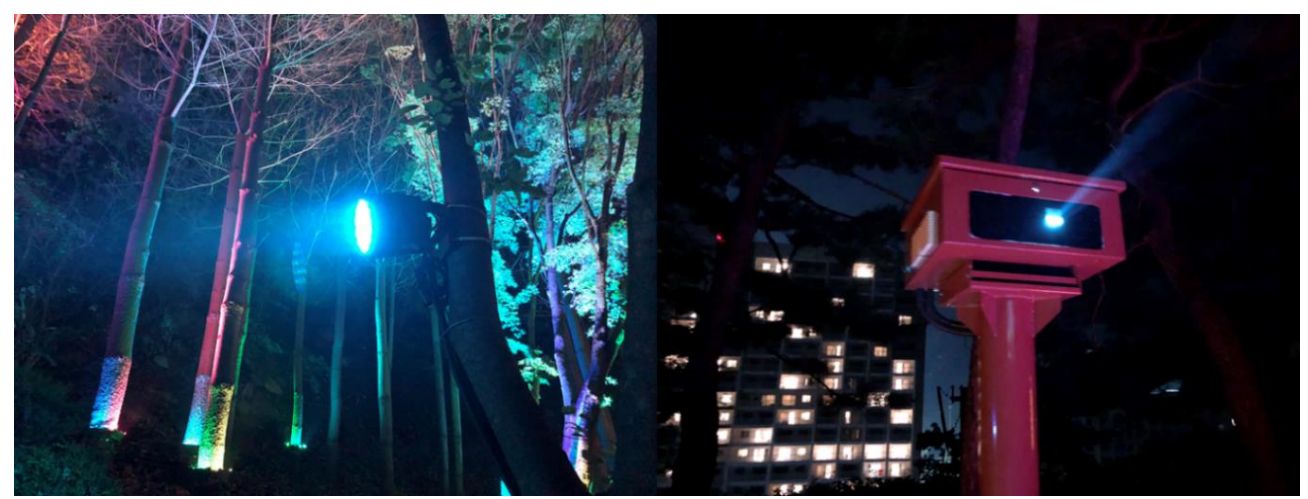

Figure 7. Self-type and Cover type of lighting devices.

The second is material and function. The functions of materials needed to be installed in outdoor spaces include waterproof, dustproof, and anti-temperature humidity. Using corrosion resistant materials, the shield function has waterproof function, and dustproof function is required in areas such as heating fans, lens parts, and connecters. You can use filters or see examples of resolution by means of shielding. Third is the maintenance of anti-temperature humidity. Lighting has its own heat, and electronic devices need a device that can maintain the temperature because they are vulnerable to moisture, high or very low temperatures. 


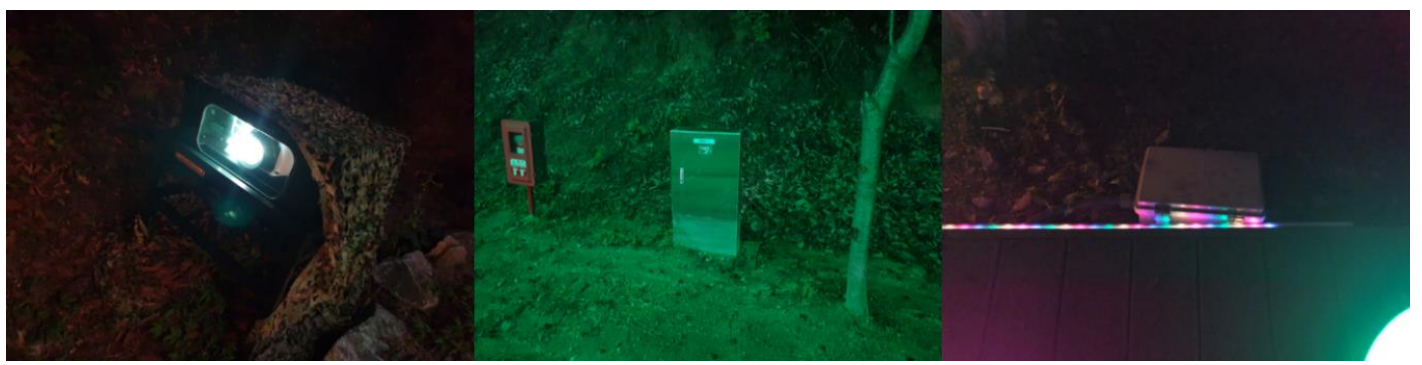

Figure 8. camouflage projector cover and exposed control box

Projector housings installed in Korea use shielded enclosures with only cooling fans as shown in Figure 8. The outer cover also uses camouflage lights or comes out of nature in a heterogeneous color. Figure 9 shows the external projector enclosures currently on the market. Coolers, heaters, and humidity control functions and materials in front projector windows have similar functions. Unfortunately, according to the case study, when artificial elements were added to the natural environment, there were no forms designed to match nature. The overseas cases were rather open without cover, and there were many types of housing that emphasized stability and efficiency in the country where the weather and the four seasons were easily changed, but only traces of military camouflage were seen trying to blend in harmony with nature. These areas remain the parts that can easily improve the quality of the content experience if designed and constructed with a little more detail.
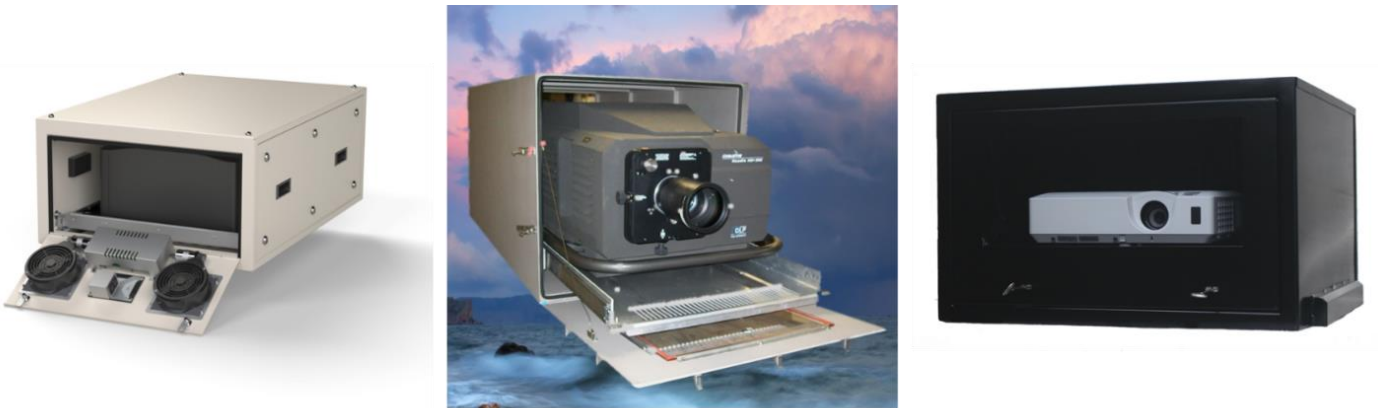

Figure 9. TEMPEST`s small \& large enclosures and ProEnc`s Outdoor Enclosure[10,11]

The third is the ease of maintenance. When shielding elements are strengthened for waterproof, dustproof, and anti-temperature humidity, maintenance is often difficult to produce. Although outdoor lighting fixtures are designed to cope with changing nature, consideration in the design part of lighting fixtures is also important as maintenance is always required. Existing outdoor connectors or equipment for special lighting are large in size and lack aesthetic design elements for maintenance. Typical examples are control boxes and numerous wires. It was easy to see both at home and abroad where electric furnaces were not buried for maintenance or to reduce construction costs, and wires were exposed to the floor or among small bushes. I hope that there are more intentions to design elements that are necessary to perform the functions of electrical materials and control boxes as well as projectors to match nature as much as possible.

Several housings must be artificially installed in nature to perform certain functions. Operating the functions of lighting, screens, and interactive elements in outdoor nature costs a considerable amount of money, and errors occur in so many factors. However, this is a basic function, and for users who visit forest park theme parks for sightseeing, these artificial nature-altering factors make them uncomfortable to watch content. Night forest park theme parks are emerging in many places, and using these stage performance techniques and new cover-up methods of artificial parts, users will have to consider not only the effects but also the facilities so that they can enjoy night forest park contents with immersion.

\section{Conclusion}

Through case studies and analysis, the classification of night forest park contents and necessary techniques were studied. We looked at the technologies to be applied to the future design of forest park lighting fixtures and the elements needed to apply the existing special stage effect technology. Currently, many temporary technologies have been used to apply many technologies, but it is expected that more outdoor and durable designs will be needed in the future.

\section{Acknowledgements}

This research is supported by Ministry of Culture Sports and Tourism(MCST) and Korea Creative Content Agency(KOCCA) in the Culture Technology(CT) Research \& Development Program 2020. 


\section{References}

1. Li, C. W., M. M. Benjamin and G. V. Korshin. (2000) Use of UV spectroscopy to characterize the reaction between NOM and free chlorine. Environmental Science and Technology 34(12), 2570-2575.

2. Benjamin, M. M. (2002) Water Chemistry. $1^{\text {st }}$ Ed. McGraw-Hill, New York, chapter 3.

3. Li, C. W., C. H. Chiu, Y. H. Lee and Y. M. Chen. (2008) Integration of ceramic membrane and compressed air-assisted solvent extraction (CAXE) for Cr(VI) recovery. Proc. of 2008 IWA North American Membrane Research Conference, Amherst, USA, 101-108.

4. Pollard, A. (1978) Flow in Tee Junction. Ph. D. Desertation, University of London, UK.

5. Kareem, A and C. M. Cheng. (1984) Across wind response of towers and stacks of circular cross section. Department of Civil Engineering, University of Houston, Report No. UHCE84-6, Houston, TX, USA.

6. Yang, L. J. (2011) US Patent 8,033,499B2.

7. Information on http://www.tku.edu.tw

8. Emanuele Giordano. (2018) Outdoor lighting design as a tool for tourist development. the case of Valladolid. European Planning Studies Aug 26(1), 55-74.

9. SY Song, DJ Shin, JY Kim. (2016) A Research on Characteristics of Stage Lighting Directing through Home/Foreign Case Analysis. Journal of the Korea Institute of Spatial Design Oct 11(5), 119-128.

10. YS Kim, ES Song, SD Hong. (2019) Examination of LED Lighting and Lighting Control System for Night Media Theme Park. TEST Engineering \& Management Nov 81, 99-105.

11. Information on https://momentfactory.com

12. Information on https://www.sonataoflight.co.kr/

13. https://www.sonohotelsresorts.com/daemyung.vp.leisure.05_03_13.ds/dmparse.dm?fbclid=IwAR0juP6 fSrQn2lXhdhz6nA96yDDwW_fNOnLDZPBuqxx6cEvzWpsv6-_W1Zo

14. Park JH, Kim JB, Kim JD. (2018) A study on Media facade as an expression of corporate identity. Journal of Korean Society of Design Science Oct 23(4), 41-52.

15. Fouquet L. (2017) Un feu digital dans une ruche multimédia : MOMENT FACTORY. ETC MEDIA Summer 111, 24-29.

16. Brandi Smith, Jeffrey Hallo. (2019) Informing good lighting in parks through visitors' perceptions and experiences. International Journal of Sustainable Lighting. Dec 21(2), 47-65. DOI:https://doi.org/10.26607/ijsl.v21i02.93.

17. Information on https://www.tempest.biz/products/outdoor-projector-enclosures/

18. Information on http://proencltd.com/ 